\title{
Progressive frontal gait disturbance with atypical Alzheimer's disease and corticobasal degeneration
}

\author{
M N Rossor, P J Tyrrell, E K Warrington, P D Thompson, C D Marsden, P Lantos
}

The National Hospital for Neurology and Neurosurgery, Queen Square, London, UK M N Rossor

P J Tyrrell

E K Warrington

C D Marsden (deceased)

MRC Cyclotron Unit, Hammersmith

Hospital, 150 Du Cane Road, London, UK

P J Tyrrell

P D Thompson

Department of Pathology, Institute of Psychiatry, De

Crespigny Park, London, UK

P Lantos

Correspondence to: Professor M N Rossor, Dementia Research Group, The National Hospital for Neurology and

Neurosurgery, Queen Square, London, WC1N 3BG, UK. Telephone 0044 171829 8773; fax 0044171 2090182.

Received 7 August 1998 and in revised form

14 December 1998

Accepted 18 March 1999

\begin{abstract}
Objectives-The clinical neuropsychological, neuroradiological, and neuropathological description of two patients presenting with a frontal gait disturbance. Methods-Clinical case note review, neuropsychological assessment, functional imaging with ${ }^{15} \mathrm{O}_{2}$ and ${ }^{18} \mathrm{~F}$-fluorodopa PET, and neuropathology.

Results-Both patients presented with frontal gait impairment and only later developed more widespread cognitive impairment. In both cases ${ }^{15} \mathrm{O}_{2}$ PET disclosed focal hypometabolism in the medial frontal lobes and in one patient ${ }^{18} \mathrm{~F}$-fluorodopa uptake into the caudate and putamen was normal. The neuropathological examination in one patient showed Alzheimer's histopathology together with large swollen eosinophilic neurons characteristic of corticobasal degeneration, which were particularly prominent in the medial frontal lobes.

Conclusion-Focal degeneration of the medial frontal lobes may present as an isolated gait disturbance and should be considered in the differential diagnosis of patients who present without an obvious structural abnormality on neuroimaging. (F Neurol Neurosurg Psychiatry 1999;67:345-352)
\end{abstract}

Keywords: progressive frontal gait disturbance; Alzheimer's disease; corticobasal degeneration

The impairment of gait that accompanies lesions of the frontal cortex and its connections may take various forms. ${ }^{1}$ Different authors have variously focused on ataxic elements, ${ }^{2}$ dysequilibrium, ${ }^{3}$ apraxia, ${ }^{45}$ and parkinsonism. ${ }^{67}$ In cases where the precise location of pathology was known, damage to the medial frontal cortex, particularly if bilateral, was associated with the greatest disturbance of gait. ${ }^{8}$ Some of the reports of gait apraxia or frontal dysequilibrium were associated with dyspraxia in the upper limb-for example, cases 1,2, and 5 of the series reported by Meyer and Barron, ${ }^{5}$ although the association is variable and severe gait disturbance with inability to stand may be seen without any evidence of upper limb dysfunction.

Most cases of gait disturbance with frontal lobe pathology have involved tumours or infarction in the territory of the anterior cerebral artery. The disturbance of gait in normal pressure hydrocephalus probably also relates to abnormalities of the frontal lobe and its connections which run adjacent to the frontal horns of the lateral ventricle. ${ }^{9}$ Cases of gait disturbance due to degenerative disease affecting the frontal lobes are only rarely reported. Meyer and Barron in the discussion of their paper refer to presenile and senile dementia as causes of apraxia of gait but give no specific examples. ${ }^{5}$ Case 3 of Petrovici, ${ }^{10}$ was interpreted as degenerative with atrophy shown on the air encephalogram, and was diagnosed as a case of Pick's disease but no histology was available. More recently, it has been suggested that focal cortical degeneration may be responsible for "gait ignition failure" on the basis of focal inferior frontal hypometabolism on PET. ${ }^{7}$

We describe two patients with early prominent gait disturbance in association with subtle dyspraxia of the upper limb; cognitive impairment developed at a later stage but remained mild in one. There was evidence of cortical atrophy on structural imaging and ${ }^{15} \mathrm{O}_{2}$ PET disclosed evidence of hypometabolism in the medial frontal lobes bilaterally. One case which came to necropsy had senile plaques and neurofibrillary tangles of Alzheimer's disease together with swollen eosinophilic neurons throughout the cortex and basophilic inclusions in the substantia nigra indicative of corticobasal degeneration. The density of eosinophilic neurons was maximal in the medial frontal lobes.

\section{Methods}

POSITRON EMISSION TOMOGRAPHY ${ }^{15} \mathrm{O}_{2}$ steady state PET was performed on the CT1/931/08/12 (CTI Knoxville, TE, USA) scanner at the MRC Cyclotron Unit at the Hammersmith Hospital, to obtain regional values of $\mathrm{CMRO}_{2}$ (cerebral metabolic rate for oxygen). The performance characteristics of this scanner, the scanning procedure, and comparative control group have been described previously. ${ }^{11-14}$ Ethical approval for these studies and those on nine volunteers was obtained from the ethics committees of the National and Hammersmith Hospitals and approval to administer radiolabelled gases was obtained from ARSAC (Administration of Radioactive Substances Advisory Committee of the United Kingdom)

Anatomical localisation of the image was obtained with reference to the atlas of Talairach et $a .^{15}$ All images were realigned to be parallel with the AC-PC (inter-commissural) line. ${ }^{16}$ The width, length, and height of each brain were used to transform the stereotactic coordinates from the anatomical atlas on to the appropriate regions in the physiological images, thus permitting anatomical identification of the focal physiological deficits. The numerical data were obtained using a circular region 
Regional $\mathrm{CMRO}_{2}(\mathrm{ml} / \mathrm{min} / \mathrm{dl})$ values for cases 1 and 2 compared with a group of normal controls (mean age ( SD) $69.3(8.6) ; n=9)$

\begin{tabular}{llll}
\hline & $\begin{array}{l}\text { Normal } \\
\text { mean (SD) }\end{array}$ & Case 1 & Case 2 \\
\hline Left cingulate gyrus & $3.22(0.52)$ & $2.08^{\star}$ & $1.94^{\star}$ \\
Right cingulate gyrus & $3.33(0.68)$ & $1.93^{\star}$ & 2.05 \\
Left F1 & $2.92(0.27)$ & $2.25^{\star}$ & $1.92^{\star}$ \\
Right F1 & $2.96(0.36)$ & $2.17^{\star}$ & $1.75^{\star}$ \\
Left F2 & $2.71(0.31)$ & 2.20 & $1.95^{\star}$ \\
Right F2 & $2.66(0.36)$ & 2.09 & 2.07 \\
Left F3 & $2.67(0.37)$ & 2.21 & 1.97 \\
Right F3 & $2.73(0.39)$ & 2.14 & 2.20 \\
Left anterior temporal & $2.74(0.40)$ & 2.60 & 2.26 \\
Right anterior temporal & $2.76(0.41)$ & 2.75 & 2.56 \\
Left posterior temporal & $2.78(0.42)$ & 2.22 & 2.34 \\
Right posterior temporal & $2.79(0.42)$ & 2.22 & 2.18 \\
Left anterior parietal & $2.84(0.42)$ & 2.74 & 2.28 \\
Right anterior parietal & $2.84(0.42)$ & 2.81 & 2.45 \\
Left posterior parietal & $2.85(0.40)$ & 2.30 & 2.19 \\
Right posterior parietal & $2.86(0.41)$ & 2.18 & 2.21 \\
Left caudate & $3.55(0.63)$ & 3.42 & 3.05 \\
Right caudate & $3.58(0.62)$ & 3.28 & 3.16 \\
Left putamen & $3.52(0.66)$ & 3.85 & 3.28 \\
Right putamen & $3.54(0.65)$ & 3.77 & 3.67 \\
Left thalamus & $3.47(0.58)$ & 3.00 & 3.15 \\
Right thalamus & $3.49(0.61)$ & 3.12 & 3.47 \\
Left occipital & $3.49(0.62)$ & 2.98 & 3.01 \\
Right occipital & $3.48(0.61)$ & 2.87 & 2.99 \\
Left cerebellum & $3.72(0.43)$ & 3.41 & 3.42 \\
Right cerebellum & $3.64(0.55$ & 3.22 & 3.12 \\
\hline
\end{tabular}

$\mathrm{F} 1=$ anterior frontal gyrus; $\mathrm{F} 2=$ middle frontal gyrus. $\mathrm{F} 3=$ posterior frontal gyrus; $\mathrm{T} 1=$ superior temporal gyrus; $\mathrm{T} 2=$ middle temporal gyrus; T3 = inferior temporal gyrus; $\mathrm{P} 1=$ post central gyrus, $\mathrm{P} 2$ = inferior parietal lobe. Indicates values which are outside two SD of the normal values (outside $95 \%$ confidence interval).

of interest, diameter $8 \mathrm{~mm}$, to maximise signals. Values for $\mathrm{CMRO}_{2}$ were obtained for each of the regions shown in the table from each of the 18 slices of the Talairach atlas on which the anatomical areas were displayed. The values for each individual patient were compared with normal values and those lying outside the $95 \%$ confidence interval $(95 \% \mathrm{CI})$ $(p<0.05)$ were considered statistically significantly different and are indicated with an asterisk.

The principal areas of hypometabolism were in the medial frontal cortex and therefore regions of interest were placed in the anterior, middle, and posterior frontal gyri and cingulate gyri. Readings were taken from those scan planes which corresponded precisely to the anatomical reference planes and thus involved sampling from more than one scan plane; the values given are the average of the appropriate number of regions of interest. In addition the anterior and posterior parietal, temporal, and occipital lobes were sampled. Basal ganglia were also sampled from the caudate, putamen, and thalamus and measurements were also made from the cerebellum.

The nigrostriatal dopamine system was assessed by measuring ${ }^{18} \mathrm{~F}$-labelled fluorodopa uptake as previously described. ${ }^{17}{ }^{18} \mathrm{~F}$-Dopa was injected intravenously at the start of scanning, and serial 5 minute scans performed up to 90 minutes after injection. Arterial blood samples taken over the same period provided measures of plasma radioactivity. No attempt to correct plasma activity for ${ }^{18} \mathrm{~F}$-dopa metabolites was attempted. Mean decay corrected counts at each time point were obtained from caudate and putamen in two planes, midfrontal cortex, occipital cortex, and cerebellum, and tissue time-activity curves were generated. Multiple time graphical analyses ${ }^{18}$ were performed using the tissue counts to obtain values for influx rate constants $(\mathrm{Ki})$ into tissue. Cerebellar values were used to generate the non-specific reference input function.

\section{CASE HISTORIES}

Patient 1

The patient was first seen as an outpatient in 1987 at the age of 70 years, when her only complaint was of difficulty walking. One year earlier she had been in an aircraft which was at risk of a crash landing. The plane had circled for 3 hours and on landing she had great difficulty in walking across the tarmac. After this she became frightened of going up and down stairs and then experienced anxiety even with walking on level ground. There was no significant medical or family history and no history of head trauma; she was taking $10 \mathrm{mg}$ temazepam at night. On examination the patient had been noted to walk with small steps as if afraid of falling and to be unable to stand with her feet together. No other abnormality had been detected.

When seen 1 year later, 2 years after the onset of walking difficulty, a marked deterioration was apparent. She was unable to walk without assistance and was confined to a wheelchair. When she was assisted to her feet, she complained of a fear of falling, and on attempting to walk she would look at her feet, which at times became crossed. When seated in a chair she would hold the arms with a tight grip. Her writing and speech output were normal and she was able to feed herself but this was at times messy. She had prominent upper limb dyspraxia, being unable to copy simple hand postures or movements. She had difficulty with miming and if successful, tended to use body parts as object. She was however, able to select the correct movement when performed by the examiner. She was emotionally rather labile. Cranial nerves were normal apart from slowness of tongue protrusion and impairment of voluntary saccades. She had bilateral grasp reflexes and a positive palmomental reflex. Her reflexes were all brisk but the plantar responses were flexor and power was considered to be normal.

This patient was first assessed neuropsychologically on her admission in September 1988. On the WAIS she obtained a verbal IQ of 78 and a performance IQ of 72 (her performance IQ was depressed by her failing to score on the block design subtest). Her recognition memory for faces was weak (36/50) and her recognition memory for words was defective $(31 / 50)$. She scored below the 5 th percentile on the graded difficulty naming test $(5 / 30) \cdot{ }^{19}$ Her perception was impaired; she scored below the 5th percentile on fragmented letters and unusual views. On the coloured progressive matrices she scored 12/36 and on the advanced progressive matrices she obtained the defective score of $1 / 12$. She failed the Weigl sorting test. In summary, 2 years after the onset of a profound gait disturbance, there was evidence of a moderate degree of impairment in all cognitive 
skills with the exception of her visual memory, which was relatively preserved. Her constructional skills were markedly impaired.

Brain CT showed mild atrophy but no other abnormality and chest radiography was normal. The patient was unable to tolerate MRI. Routine haematology and biochemistry, which included Vitamin $\mathrm{B}_{12}$ and thyroid function and negative treponemal serology,were normal. An EEG showed modest intermediate slow wave frequencies in the temporal region but no other abnormalities. Examination of the CSF disclosed a protein of $0.57 \mathrm{~g} / 1$ but no cells. ${ }^{15} \mathrm{O}_{2}$ PET was performed 2 years after onset when the patient was not taking medication. The patient was subsequently lost to follow up.

Patient 2

A 69 year old right handed retired woman was admitted with a history of mild impairment of gait with occasional falls after arthroscopy for a painful right knee. Her gait impairment was characterised by unsteadiness and difficulty initiating movement of the feet. She tended to fall backwards and her first fall occurred in a golf bunker while swinging her golf club. The unsteadiness and tendency to fall backwards became increasingly severe. There was no relevant medical or family history and no history of head trauma. Initial examination did not show obvious neurological deficit and investigations included a normal CT. She was given a trial of levodopa, antidepressant drugs, and steroids without benefit; repeat CT raised the possibility of a small ischaemic area and she was therefore anticoagulated for a short period on the basis that her gait impairment might be due to multiple infarctions. She had also attended a psychologist for treatment of a presumed "space phobia".

When first seen at the National Hospital in 1986 (3 years after onset) she had considerable difficulty getting out of a chair and walking but was able to do so unaided. There was a tendency to fall backwards and she became extremely anxious at the prospect of standing and walking. In addition, her handwriting was becoming less legible and she had occasional problems with manipulating buttons. According to her husband she was also less able to make decisions.

Examination on the first admission showed a wide based shuffling gait with the use of a stick. Tone was increased in all limbs and was thought to be paratonia or Gegenhalten rather than extrapyramidal rigidity. She had a positive glabella tap and positive palmomental and grasp reflexes; the remainder of the examination was unremarkable. Psychiatric assessment showed no evidence of depression. She was assessed on the WAIS (November 1986) and obtained a verbal IQ of 100 and a performance IQ of 106. On the NART she obtained an IQ equivalent score of 110 , indicating that her optimum level of functioning was only marginally higher than documented.

Brain CT was considered normal and MRI showed minimally increased signal around anterior parts of the lateral ventricles only. The EEG was of low amplitude but otherwise nor- mal. Protein concentration in CSF was 0.24 $\mathrm{g} / \mathrm{l}$, there were no cells, and oligoclonal bands were negative. Routine biochemistry and haematology including $\mathrm{B}_{12}$ and syphilis serology were all normal.

When reviewed only 4 months later she was unable to walk and would sit in a chair gripping the sides from fear of falling. Knitting and writing had become rather difficult but she was playing bridge successfully although experiencing some difficulty with manipulating the cards. Examination showed that she was able to stand with the help of two people but had great difficulty initiating very small wide based steps with a tendency to fall backwards. There was marked Gegenhalten in all limbs. Reflexes were symmetric with flexor plantar responses. A repeat CSF examination was normal as were sensory evoked potentials. Conduction in corticomotor neuron pathways to legs and arms, assessed by the latency of EMG responses in the upper and lower limbs to electrical brain stimulation, were normal. She was reassessed on the WAIS. There had been no decrement in her verbal IQ (101) and only a mild decline in her performance IQ (96). Her performance on a verbal recognition memory test was normal $(47 / 50)$.

Six months later she was entirely chairbound and had noticed deteriorating hand function such that she required help with dressing, cutting up food, and doing up buttons and she held a cup with two hands. She also thought that her concentration was poor. There was no history of urinary incontinence. On examination she was unable to stand and if supported her legs would shake. She was able to perform simple foot movements such as tapping and was able to make some bicycling and stepping movements when seated, by contrast with the great difficulty in making any leg movements when standing. Her facial expression and rate of spontaneous blinking were normal, as was her speech. There was some impairment of voluntary horizontal saccades when the patient was instructed to look to the right and the left, which were slow although performed accurately. Tone was increased in axial muscles and she was unable to turn over when lying in bed. She had positive grasp and pout reflexes and had definite evidence of dyspraxia both in miming hand postures and transitive and intransitive hand movements.

At this time (October 1987) there had been a further decrement in her intellectual competence (verbal IQ 91, performance IQ 82). However, her performance on recognition memory tests was very satisfactory (recognition memory test for words $48 / 50$ and recognition memory for faces 44/50). Her language skills were normal (GNT 25/30).

Nine months later she had gained weight and had seen a psychiatrist 2 months earlier for depression. Amitriptyline was started but resulted in a tremor. Examination again showed slowness of horizontal voluntary saccades with some impersistence of pursuit and mild limb tremor. The remainder of the examination was unchanged. ${ }^{15} \mathrm{O}_{2}$ PET and neuropsychological assessments were performed on 


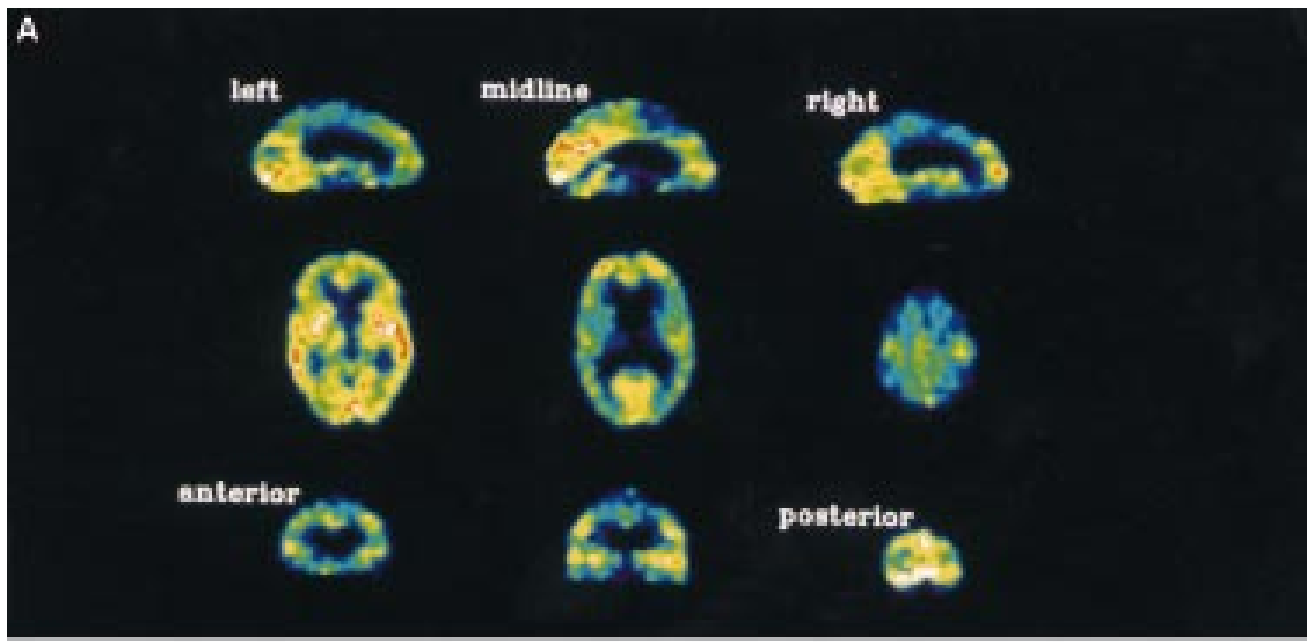

B

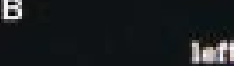

left.

miditine
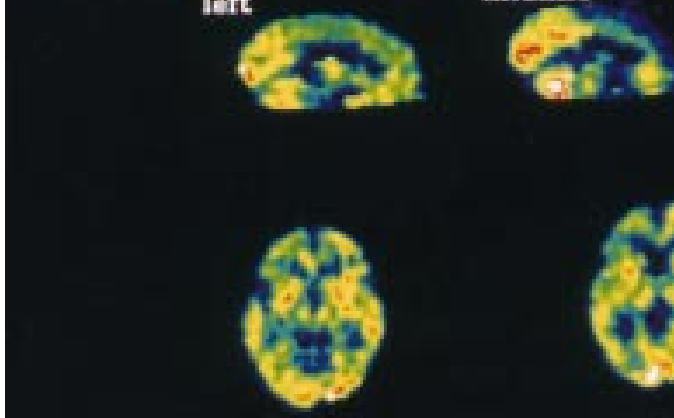

right

rest

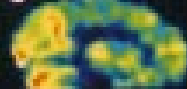

anterior
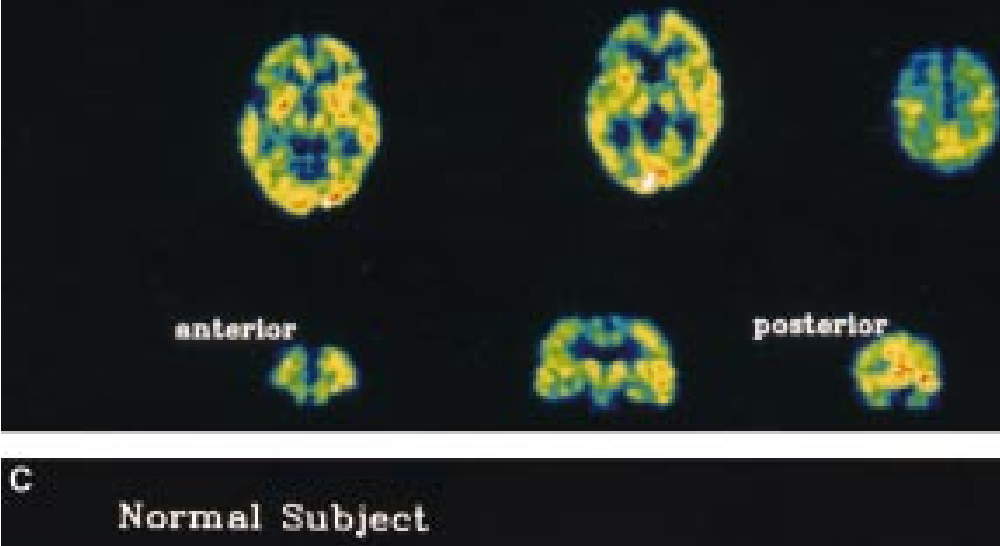

posterior
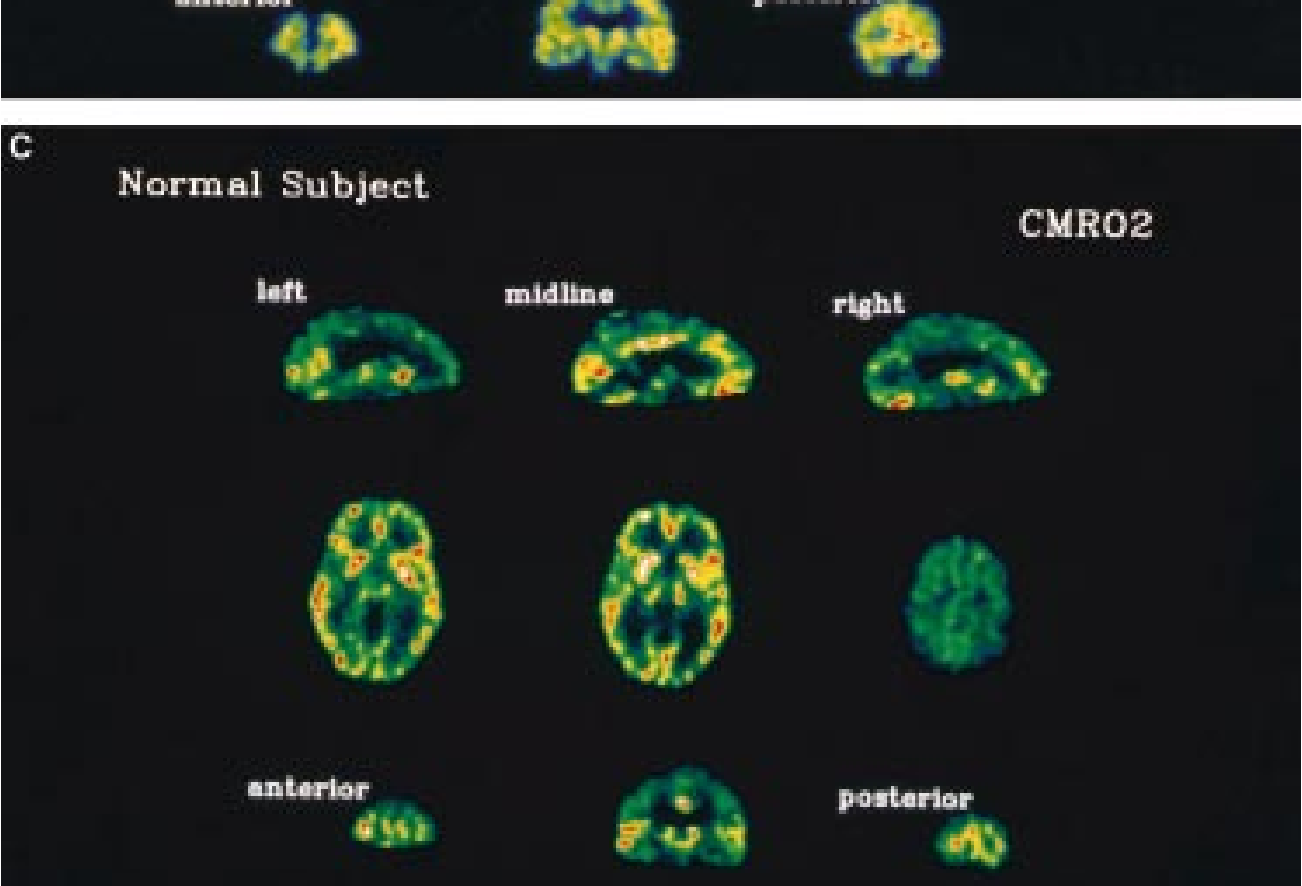

Figure 1 (A) $\mathrm{CMRO}_{2}$ images from patient 1 showing sagittal, transverse, and coronal slices taken at the same levels as the normal subject. Marked midfront hypometabolism is seen on the midline sagittal slice, the most superior transverse slice, and the anterior coronal slice. The frontal pole is to the right in the sagittal images. (B) $\mathrm{CMRO}_{2}$ images from patient 2 showing sagittal, transverse and coronal slices taken at the same level as the normal subject. Marked midfrontal hypometabolism is seen on the midline sagittal slice, the most superior transverse slice, and the anterior coronal slice. The frontal pole is to the right in the sagittal images. (C) $\mathrm{CMRO}_{2}$ images from a representative normal subject. Sagittal slices are shown in the top row, transverse slices in the middle row, and coronal slices in the bottom row. Sagittal slices are taken through the left thalamus, the midline, and the right thalamus. Transverse slices are taken at the level of the basal ganglia, the thalami, and $7.0 \mathrm{~cm}$ above the orbitomeatal line through the frontal lobes. Coronal slices are taken through the occipital cortex. The frontal lobe is to the right in the sagittal images. 


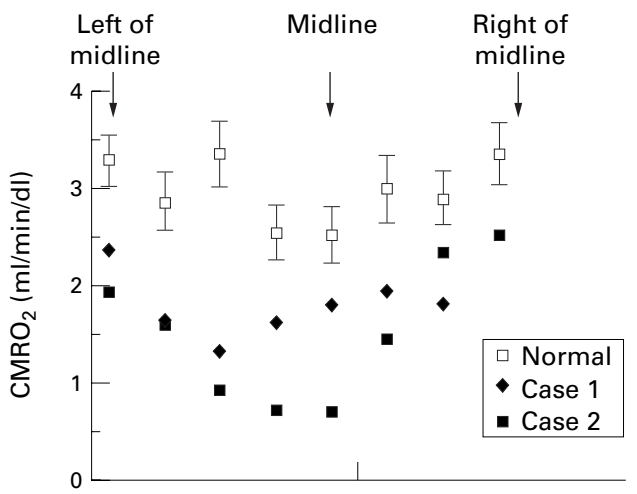

Figure 2 Regions of interest, radius 2 pixels, were drawn on adjacent sagittal sections of the $\mathrm{CMRO}_{2}$ scans of patient 1 and 2, and the normal subjects. The first region of interest was drawn on the cortical ribbon immediately superior to the thalamus, and continued on adjacent sagittal slices to the level of the right thalamus. The error bars show the SD of the normal values around the mean. The figure shows the marked reduction in $\mathrm{CMRO}_{2}$ in the midline sagittal sections, in both patients, also visible on the scans.

this admission when the patient was on no medication. Her scores on the WAIS-R were VIQ 94 and PIQ 78, (failing to score on the block design subtest). Her performance on recognition memory tests and naming remained satisfactory.

During the next year there was a more rapid deterioration requiring full time nursing care, and 18 months later she died from bronchopneumonia some 7 years after her first symptoms.

POSITRON EMISSSION TOMOGRAPHY

Cerebral blood flow $(\mathrm{CBF})$ and oxygen extration rate data showed coupled changes in flow with a constant oxygen extraction which did not differ from normal; therefore, $\mathrm{CMRO}_{2}$ data alone are given. Both cases showed symmetric areas of hypometabolism medially in the frontal lobes with marked reduction in the anterior frontal gyrus and cingulate gyrus. Sampling in these areas provided values which were outside the $95 \%$ CIs for the normal group. Only medial frontal cortical areas showed a statistically significant reduction in $\mathrm{CMRO}_{2}$ although there was a non-significant trend towards lower values in some temporal and parietal areas. The values are shown in the table. Figure $1 \mathrm{~A}$ and $\mathrm{B}$ show representative scans from patients 1 and 2 respectively and figure $1 \mathrm{C}$ shows a normal subject for comparison. Figure 2 illustrates the results graphically.

The influx constants $(\mathrm{Ki})$ for ${ }^{18} \mathrm{~F}$-dopa were within the normal range: mean caudate 0.01086 , mean putamen 0.00985, (normal $\mathrm{n}=7$; caudate 0.01040 (SD 0.00046), putamen 0.01060 (SD 0.00088)).

\section{NEUROPATHOLOGY}

Patient 2

Blocks of tissue were taken from the frontal, temporal (with the hippocampus and thalamus), parietal, and occipital lobes, basal ganglia (at two levels), cerebellar vermis and hemisphere, midbrain, pons, and medulla oblongata. Altogether, 20 samples were taken and embedded in paraffin wax for neurohistology.
Sections were stained with haematoxylin and eosin, Luxol fast blue and cresyl violet, and Congo red; and impregnated with silver according to Gallyas, and Marsland and Glees. The following antibodies were used in the avidin-biotin complex reaction (Amersham, UK): ubiquitin (DAKO, High Wycombe, UK), tau protein (Sigma, Poole, UK), GFAP (DAKO), $\alpha$ B-crystallin (Novo-castra), and $\beta A 4$ protein (12-28 fragment, kindly provided by Professor BH Anderton, Institute of Psychiatry, London).

The fixed brain weighed $1074 \mathrm{~g}$ and the brainstem and cerebellum $169 \mathrm{~g}$. There was marked bilateral atrophy of the convexities of the brain. The sulci were widened, particularly in the frontoparietal region. The large cerebral arteries showed many atherosclerotic plaques with $80 \%$ occlusion of the left middle cerebral artery at its origin. The atrophy of the frontoparietal and temporal regions was confirmed on coronal slices. However, the ventricular system was only slightly dilated. The hippocampus was normal on both sides and filled the temporal horn. The globus pallidus on both sides was darker than usual and there was a yellowish slit-like, ragged edged cavity of $1.0 \mathrm{~cm}$ along the longest axis in the left corpus striatum. The white matter of the centrum semiovale seemed firm. The substantia nigra and locus coeruleus were well pigmented. The brainstem and the cerebellum were normal.

Histology showed many large swollen eosinophilic neurons, sometimes apparently without nuclei, throughout the neocortex (fig $3 \mathrm{~A}$ ). These cells were most numerous in the superior and medial aspect of the frontal lobe, the supplementary motor area being particularly severely affected. These swollen cells were best demonstrated by $\alpha \mathrm{B}$-crystallin (fig $3 \mathrm{~B}$ ), but they were negative for tau protein although occasional cells showed faint peripheral staining of the cytoplasm. With antibody to ubiquitin there was an equivocal granular, cytoplasmic staining. Several neurons were intensely eosinophilic with well defined, sometimes angular contours, whereas a few others were frankly pyknotic. Astrocytosis was demonstrated by GFAP. Alzheimer type changes, both neurofibrillary tangles and senile plaques, were abundant throughout the neocortex; both were most numerous in the occipital cortex. Tau immunostaining showed, in addition to neurofibrillary tangles and neuritic plaques, neuronal inclusions in the superficial cortex, so called astrocytic plaques (fig $3 \mathrm{C}$ ), and oligodendroglial coiled bodies. Immunocytochemistry for $\beta \mathrm{A} 4$ protein showed, in addition to plaques, subpial and diffuse deposits. The hippocampus was well preserved: only occasional granulovacuoles, a few tangles, a couple of plaques and several Hirano bodies were seen. Histology confirmed the presence of an old infarct in the left caudate nucleus. The globus pallidus showed neuronal loss, pigment containing macrophages, and astrocytosis. The nucleus basalis of Meynert was somewhat depleted and an occasional tangle was noted. The substantia nigra showed striking cell loss, extraneuronal pigment, several basophilic inclusions (fig $3 \mathrm{D}$ ), 

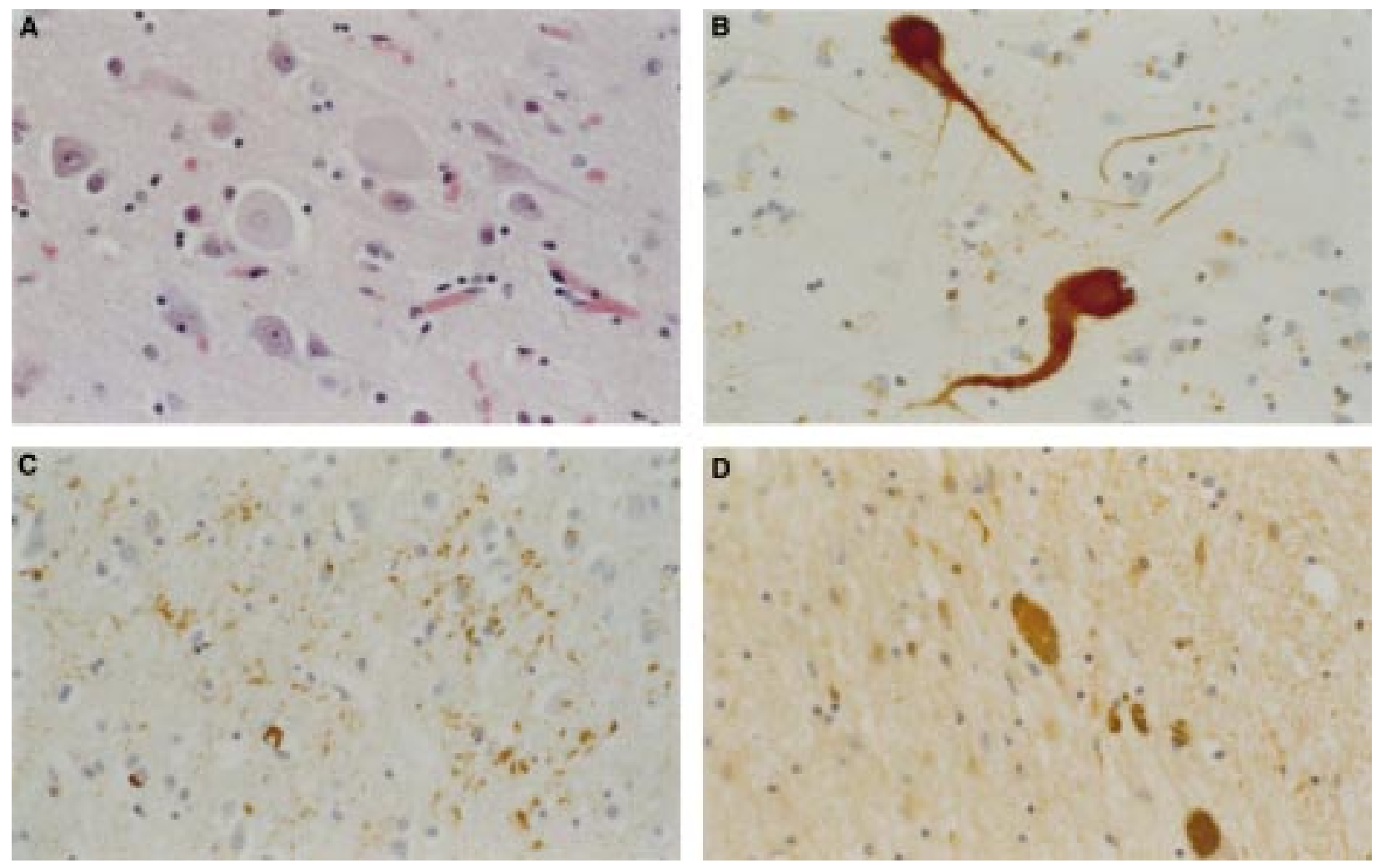

Figure 3 (A) Swollen, achromatic neurons in the frontal cortex (haematoxylin and eosin). (B) The abnormal cells are positively stained with an antibody to aB-crystallin. (C) Tau-positive inclusions in the small neurons and astrocytic processes in the superficial frontal cortex. (D) Neuronal loss, extraneuronal pigment, and a tau positive inclusion in a pigmented neuron (centre) of the substantia nigra. $B$ and D immunohistochemistry: ABC method. $A$ and $C$ originally $\times 120$.

an occasional Marinesco body, and astrocytosis. Extraneuronal pigment was also seen in the locus coeruleus. Lewy bodies were not present either here or in the substantia nigra. There was an occasional tangle in the medulla oblongata. The cerebellum showed small infarcts and occasional axonal swellings (torpedoes) of the Purkinje cells.

Blood vessels had somewhat thickened fibrous walls, some perivascular mononuclear cells, and calcification of the vascular wall in the lentiform nucleus. Amyloid was demonstrated by Congo red and $\beta \mathrm{A} 4$ protein in the blood vessels, both in the meninges and in the cerebral parenchyma.

\section{Discussion}

Two patients are presented with the common feature of a profound gait disturbance in association with upper limb dyspraxia. Initially the arm dyspraxia was only apparent on specific testing but in both patients there was subsequent development of clumsiness. The early gait disturbance was accompanied by a prominent fear of falling and as the disease progressed both patients became chairbound. Even sitting near the edge of the bed resulted in considerable anxiety and both patients characteristically sat in chairs holding the arms tightly. The early features of anxiety and the paucity of neurological abnormalities resulted in initial psychiatric diagnoses in both cases and as a result treatments for anxiety, depression, and "space phobia" were instituted. This was particularly relevant in patient 1 in whom the initial presentation was an acute gait disturbance in a setting of extreme anxiety when the patient was involved in a near plane crash. It was only the subsequent development of a progressive course that led to the diagnosis of an organic neurological disturbance. The clinical course was of progression to more widespread cognitive impairment after about 2 years in the first patient but in the other progression was very slow and cognition remained relatively preserved. It is notable that urinary incontinence was not a feature in either of these patients.

Apart from the gait disturbance neurological examination disclosed little abnormality at presentation. Increased muscle tone was apparent later, particularly in the legs, at the time of the development of upper limb apraxia. It was thought, however, that this had the characteristics of Gegenhalten which may be found in association with dyspraxia. ${ }^{20}$ There were no cerebellar signs, prominent extrapyramidal features, or pyramidal signs. Grasp and pout reflexes were elicited but utilisation behaviour was not apparent. Conduction in the central motor pathways was normal in the one patient examined, suggesting that the corticomotor neuron connections with leg muscles were normal, and could not therefore account for the walking difficulty.

The clinical and neurological features were indicative of frontal lobe disturbance and although the structural imaging available showed no obvious abnormalities, PET demonstrated hypometabolism in the medial 
frontal cortex; the eosinophilic swollen neurons in patient 2 were also maximal in medial frontal lobes. The normal fluorodopa scan supports the view that the abnormality cannot be attributed to depletion of dopamine in the nigrostriatal system and there was no evidence of Lewy body formation in the substantia nigra.

The evidence for involvement of the frontal lobes in abnormal gait is primarily clinical. Particular emphasis has been placed on medial frontal lesions, ${ }^{51}$ which is supported by the abnormalities on PET in these patients showing an area of hypometabolism encompassing the supplementary motor area. Damage to this area may also produce the alien hand sign with forced groping ${ }^{22}{ }^{23}$ but this was not apparent in these patients. Surgical lesions of the supplementary motor area may result in abnormalities of sequencing ${ }^{24}$ and in two patients with left mesial hemispheric infarcts bilateral ideomotor dyspraxia for transitive limb movements, as seen in the patients reported here, could be demonstrated. ${ }^{25}$ Moreover, in five cases of corticobasal degeneration reported by Tsuchiya et al the lesion responsible for the apraxia was suggested to be the premotor cortex. ${ }^{26}$ In addition to discrete infarcts affecting the medial hemisphere diffuse subcortical ischaemia (Binswanger's disease) may present with gait disturbance which has been attributed to damage to the afferent and efferent connections of the leg areas of the motor and premotor cortex. ${ }^{6}$

Histopathology was only available in one patient although the early clinical features were strikingly similar. The neuropathology of this case is complex. The overall histological features warrant the diagnosis of corticobasal degeneration: there were swollen achromatic cells in the cortex, corticobasal inclusions in the substantia nigra, tau positive neuronal inclusions in the superficial cortical layers, astrocytic plaques, and oligodendroglial coiled bodies all in the setting of a predominantly frontoparietal atrophy. In addition, there was Alzheimer-type pathology; neurofibrillary tangles and neuritic plaques were present both in the cortex and in the deep grey matter. However, there were two unusual features. Firstly, the hippocampus was little affected. Secondly, both plaques and tangles were most numerous in the occipital cortex. Although this case fulfils the CERAD criteria, ${ }^{27}$ it raises questions about limited sampling which, for example, leaves out the occipital cortex, the most affected area in this case. A more recent consensus report on the diagnosis of Alzheimer's disease ${ }^{28}$ recommends more extensive sampling of both the cortex to include the occipital cortex and deep grey matter. The Alzheimer's disease was accompanied by amyloid angiopathy as demonstrated by $\beta$ A4 immunohistochemistry. The blood vessels also showed atherosclerosis and there were two small infarcts. The neuropathological diagnosis therefore is corticobasal degeneration, atypical Alzheimer's disease with amyloid angiopathy, and cerebral atherosclerosis with small infarcts.

The site of maximal density of the swollen eosinophilic neurons in the medial frontal cor- tex corresponds to the region of hypometabolism demonstrated by PET and the area implicated in frontal gait disorders from clinical studies. Corticobasal degeneration is characterised as here, by swollen eosinophilic neurons and substantia nigra basophilic inclusions but not by coexistent Alzheimer changes. Clinically patients with corticobasal degeneration usually develop an asymmetric motor disorder of the upper limb with involuntary movements, myoclonic jerks, and severe apraxia. The lower limbs may be involved but usually asymmetrically. Fluorodopa imaging in patients with clinically diagnosed corticobasal degeneration shows reduced uptake in both caudate and putamen, which was not seen in our case. ${ }^{29}$ Moreover, the pattern of hypometabolism in the frontal lobe is predominantly posterior together with more extensive asymmetric involvement of the parietal and temporal cortex, corpus striatum, and thalamus. ${ }^{30-31}$

The swollen achromatic neurons of corticobasal degeneration overlap with the features of Pick's disease. The term Pick's disease has been variably used for circumscribed atrophy with or without the presence of Pick cells but disturbance of gait is not a typical feature. ${ }^{32}$ Some cases are described with prominent basal ganglia atrophy but even in these cases the reported gait disturbance is not dramatic. ${ }^{33} 34$ Case number 3 of the series of gait apraxias described by Petrovici, was reported to have Pick's disease on the basis of circumscribed atrophy seen on air encephalography, but no neuropathological confirmation was available. ${ }^{10}$ This patient differed from ours in the development of a more rapid dementia. Gait disturbance has been reported in association with Alzheimer's disease but this is not prominent at an early stage. Extrapyramidal features are more commonly found but not in association with bradykinesia or the gait disturbance of Parkinson's disease unless there is concomitant Lewy body disease. ${ }^{17}{ }^{35}$ The vascular changes found at necropsy were not apparent on structural imaging although suspected initially clinically. However, the distribution of vascular change is unlikely to be relevant.

The occurrence of a second pathology with corticobasal degeneration, although rare, has been reported. Thus, Schneider et $a l^{36}$ reported three out of 11 cases of corticobasal degeneration in which Alzheimer's disease also occurred. Ball et al also reported a patient with hemianopia and subsequent alien limb with marked asymmetric apraxia who was found to have a combination of eosinophilic swollen neurons and Alzheimer's histopathology. ${ }^{37}$ Whether such cases, including patient 2 in this report, represent a discrete disorder or cbxoexistence of two diseases remains to be seen. It is not possible to assess how common such cases are but review of the literature suggests that presentation of an isolated frontal gait disturbance on a degenerative basis is rare and this contributed to the early diagnostic difficulties. Focal neurodegeneration needs to be considered in the diagnosis of patients 
with progressive frontal gait disorder in whom imaging excludes an obvious structural lesion.

We thank Dr GD Schott, Dr RG Lascelles, and Dr NC Brown for referring these patients and providing clinical information, Mrs Heidi Barnes for technical assistance and the London Mrs Heidi Barnes for technical assistance and the London Neurodeg.

1 Nutt JG, Marsden CD, Thompson PD. Human walking and higher-level gait disorders, particularly in the elderly. Neuhigher-level gait disorders,
rology 1993;43:268-79.

2 Bruns L. Uber storungen des Gleichgewichtes bei Stirnhirntumoren. Dtsch Med Wochenschr 1892;18:138-43.

3 Van Bogaert L, Martin P. Sur deux signes du syndrome de desequilibration frontale: l'apraxie de la marche et l'atonie statique. Encephale 1929;24:11-18.

4 Gerstmann J, Schilder P. Uber eine besondere Gangstorung bei Stirnhirnerkrankung. Wschr Med Schr 1926;76:97-102

5 Meyer JS, Barron DW. Apraxia of gait: a clinicophysiological study. Brain 1960;83:261-84.

6 Thompson PD, Marsden CD. Gait disorder of subcortical arteriosclerotic encephalopathy: Binswanger's disease. Mov Disord 1987;2:1-8.

7 Atchison PR, Thompson PD, Frackowiak RS, et al. The syndrome of gait ignition failure: a report of six cases. Mov Disord 1993;8:285-92.

8 Denny-Brown D. The nature of apraxia. 7 Nerv Ment Dis 1958;216:9-33.

9 Fisher CM. Hydrocephalus as a cause of disturbances of gait in the elderly. Neurology 1982;32:1358-63.

10 Petrovici I. Apraxia of gait and of trunk movements. $7 \mathrm{Neu}$ rol Sci 1968;7:229-43.

11 Spinks T, Guzzardi R, Bellina CR. Performance characteristics of a whole body positron tomograph. $\mathcal{F} \mathrm{Nucl}$ Med 1988 29:1833-41

12 Tyrrell PJ, Warrington EK, Frackowiak RSJ, et al. Heterogeneity in progressive aphasia due to focal cortical atrophy. A clinical and PET study. Brain 1990;113:132136.

13 Frackowiak RSJ, Lenzi GL, Jones T, et al. Quantitative measurement of regional cerebral blood flow and oxygen metabolism in man using ${ }^{15} \mathrm{O}$ and positron emission tomography: theory, procedure and normal values. $7 \mathrm{Com}-$ put Assist Tomogr 1980;4:727-36. 14 Lammertsma AA, Jones T. Correction for the presence of intravascular Ax, Jo Description of the method. $f$ Cereb Blood Flow Metab 1983; 3:416-24.

15 Talairach J, Szikla G, Tournoux P, et al. Atlas d'Anatomie stereotaxique du Telencephale. Paris: Masson et Cie (GENERIC), 1967

16 Friston KJ, Passingham RE, Nutt JG, et al. Localization in pet images: direct fitting of the intercommissural (ac-pc) line. F Cereb Blood Flow Metab 1989;9:690-5.

17 Tyrrell PJ, Sawle GV, Ibanez V, et al. Clinical and positron emission tomographic studies in the "extrapyramidal syndrome" of dementia of the Alzheimer type. Arch Neurol 1990;47:1318-23.

18 Patlak CS, Blasberg RG, Fenstermacher JD. Graphical evaluation of blood-to-brain transfer constants from multiple-time uptake data. $\mathcal{F}$ Cereb Blood Flow Metab 1983; $3: 1-7$.
19 Warrington EK. Manual for the recognition memory test for words and faces. Windsor: NFER Nelson, 1984.

20 Tyrrell P, Rossor M. The association of gegenhalten in the upper limbs with dyspraxia. $\mathcal{F}$ Neurol Neurosurg Psychiatry 1988;51:995-7.

21 Denny-Brown D. The nature of apraxia. 7 Nerv Ment Dis 1958;216:9-33.

22 Brion S, Jedynak CP. Trouble du transfert interhemispherique a propos de trois observations de tumeurs du corps calleux: le signe de la main etrangere. Rev Neurol (Paris) 1972;126:257-66.

23 Goldberg G, Mayer NH, Toglia JU. Medial frontal-cortex infarction and the alien hand sign. Arch Neurol 1981;38: 683-6.

24 Laplane D, Talairach J, Meininger V, et al. Clinical consequences of corticectomies involving the supplementary motor area in man. $\mathcal{F ~ N e u r o l ~ S c i ~ 1 9 7 7 ; 3 4 : 3 0 1 - 1 4 . ~}$

25 Watson RT, Fleet WS, Gonzalezrothi L, et al. Apraxia and the supplementary motor area. Arch Neurol 1986;43:78792.

26 Tsuchiya K, Ikeda K, Uchihara T, et al. Distribution of cerebral cortical lesions in corticobasal degeneration: a clinicopathological study of five autopsy cases in Japan. Acta Neuropathol 1997;424;416-24.

27 Mirra SS, Heyman A, McKeel D, et al. The consortium to establish a registry for Alzheimer's disease (CERAD): 2 standardization of the neuropathologic assessment of Alzheimer'sdisease. Neurology 1991;41:479-86.

28 Hyman BT, Trojanowski JQ. Consensus recommendations for the postmortem diagnosis of Alzheimer disease from the National Institute on Aging and the Reagan Institute Working Group on diagnostic criteria for the neuropathological assessment of Alzheimer disease [editorial]. I Neuropathol Exp Neurol 1997;56:1095-7.

29 Sawle GV, Brooks D, Marsden CD, et al. Corticobasal degeneration; a unique pattern of regional cortical oxygen metabolism and striatal fluoro dopa uptake demonstrated by positron emission tomography. Brain 1991;114:541-56.

30 Blin J, Vidailhet MJ, Pillon B, et al. Corticobasal degeneration: decreased and asymmetrical glucose consumption as studied with PET. Mov Disord 1992;7:348-54.

31 Nagahama Y, Fukuyama $\mathrm{H}$, Turjanski $\mathrm{N}$, et al. Cerebral glucose metabolism in corticobasal degeneration: comparison with progressive supranuclear palsy and normal controls. Mov Disord 1997;12:691-6.

32 Binetti G, Growdon JH, Vonsattel JP. Pick's disease. In: Growdon $\mathrm{JH}$, et al, eds. The dementias. Newton: Butterworth-Heinemann, 1998:7-44.

33 Akelaitis AJ. Atrophy of basal ganglia in Pick's disease: a clinicopathological study. Arch Neurol Psychiat 1944;51:2734.

34 Munoz-Garcia D, Ludwin SK. Classic and generalized variants of Pick's disease: a clinicopathological, ultrastructural, and immunocytochemical comparative study. Ann Neurol 1984;16:467-80.

35 Lennox G. Dementia with Lewy bodies. In: Growdon JH, et al, eds. The dementias. Boston: Butterworth-Heinemann, 1998:67-79.

36 Schneider JA, Watts RL, Gearing M, et al. Corticobasal degeneration: neuropathologic and clinical heterogeneity. Neurology 1997;48:959-69.

37 Ball JA, Lantos PL, Jackson $M$, et al. Alien hand sign in association with Alzheimer's histopathology. 7 Neurol Neurosurg Psychiatry 1993;56:1020-3. 